\title{
China’s Precision Medicine should aim for Innovation
}

\section{Zhiyi Zhang, Chunling Li, Weiyan Wang and Yue Zhang*}

The First Clinical College, Harbin Medical University, China

*Corresponding author: Yue Zhang, The First Clinical College, Harbin Medical University, Harbin, China 150001, Tel: 86 451 8666 6421; E-mail: toronto101@163.com Received date: Jun 03, 2016; Accepted date: Jun 05, 2016; Published date: Jun 07, 2016

Copyright: () 2016 Zhang Y, et al. This is an open-access article distributed under the terms of the Creative Commons Attribution License, which permits unrestricted use, distribution, and reproduction in any medium, provided the original author and source are credited.

\section{China's Precision Medicine (CPM)}

China's Precision Medicine (CPM) illustrated the exciting potential of having the right drug for the right patient at the right time with a "more organized, more efficient" program based on high-throughput multiple-dimension Omics tool kit-produced big data including those from encompassing but not limited to, genomics, transcriptomics, proteomics, metabolomics, epigenomics, immunomics, and so on. However, CPM need aim for a better innovation and otherwise fail to achieve these goals.

CPM guidelines [1] require a project ratio between local finance and central government funding of $\geq 1$. This may work in several richer cities (e.g. Beijing and Shanghai). However, most patients in northeastern China cannot afford effective biologics and the chance of obtaining local finance is lower and thus the distribution of funding is extremely biased towards rich towns (Indeed around 95\% (170/182) of candidate institutions toward the final PM-project defense turns out from rich towns after the first initial screening) [2]. Furthermore, certain diseases (e.g. Kashin-Beck disease) are seen more often in certain locations. Although northeastern China has comparative research advantages (e.g. one single faculty in Jilin University has three top-level State key labs compared to none in some universities in rich towns), without appropriate local funding, they will miss out on CPM mega-projects. We remained lucky in this list of candidate institutions, but we finally missed the great chance in part due to this project ratio.

Greater diversity of proposals would benefit CPM through the increased potential for innovations as induced pluripotent stem cells increasing the variety of expressed genes to restore the pluripotency of cells [3]. However, CPM guidelines came out so narrowly described that many intended recipients seem somehow obvious [4]. The innovation risks being stifled by a lack of sufficiency of variety, quality and assessment equality of proposal. PM should look for better innovations and not miss cutting-edge thinking.
We may understand that infancy CPM should not be hijacked by any larger sequencing facilities and rely on urgently revolutionizing sequencing techniques to lower the cost of genome-wide sequencing for clinical practice cost-effectiveness. Addressing the "shortage of doctors" through training interdisciplinary intermediates connecting clinicians and lab-workers would help CPM innovation goals. China could also consider funding cost-effective long-term >5-year longitudinal and multicenter collaborative data-sharing spatiotemporal matrix of biology and precision medicine projects [5] (e.g. osteoarthritis genesis studies) rather than short-term 3-5 years projects only. Besides, some cost-effective projects may benefit from the researchers' dedications to science, like the discovery of DNA-guided genome editing using the Natronobacterium gregoryi Argonaute [6], for which the correspondent's lab has been supported with limited resources. Without significant changes and successes, CPM may be difficult to achieve its goals ideally.

\section{References}

1. http://www.most.gov.cn/mostinfo/xinxifenlei/fgzc/gfxwj/ gfxwj2016/201603/t20160308_124540.html

2. Development Center for Medical Science and Technology. http:// www.dcmst.org.cn/tz3/277-2016-05-06-06-57-54.

3. Takahashi K, Yamanaka S (2006) Induction of pluripotent stem cells from mouse embryonic and adult fibroblast cultures by defined factors. Cell 126 : 663-76

4. Shi Y, Rao Y (2010) China's research culture. Science 329: 1128.

5. Zhang Y (2014) Tweeting biology for better data and better connections. J Nucl Med Radiat Ther 6:1.

6. Gao F, Shen XZ, Jiang F, Wu Y, Han C (2016) DNA-guided genome editing using the Natronobacterium gregoryi Argonaute. Nat Biotechnol. 